\title{
Magnetic resonance imaging, ultrasound and magnetogastrography evaluation on the gastrointestinal system
}

\author{
Teodoro Córdova-Fraga ${ }^{1 *}$, Angel Lizbeth Piña Ruiz ${ }^{1}$, José Alfredo Soto Álvarez ${ }^{1}$, Rafel Guzman Cabrera ${ }^{2}$, Mauricio Sanchez Barajas ${ }^{3}$ and \\ José María de la Roca Chiapas ${ }^{4}$ \\ ${ }^{1}$ Departamento de Ingeniería Física-DCI, Universidad de Guanajuato campus León Loma del Bosque N. 103, Lomas del Campestre, 37150 León, GTO, Mexico \\ ${ }^{2}$ Division de Ingenierías, Universidad de Guanajuato campus Irapuato-Salamanca Salamanca-Valle de Santiago Km 3.5+1.8 Km, Palo Blanco, 36885 Salamanca, \\ GTO, Mexico \\ ${ }^{3}$ Hospital General de Zona con Medicina Familiar N. 21 - IMSS León, Coral N. 101, Colonia San Rafael, 37380 León, GTO, Mexico \\ ${ }^{4}$ Department of Psychology, Division of Health Sciences, Universidad de Guanajuato Campus León. Blvd. Puente Milenio N. 1001, León, Guanajuato CP 37670 , Mexico
}

\begin{abstract}
Evaluating the gastrointestinal tract (GI) has become vital due to the essential clinical information it presents, particularly for diabetic and elderly patients. Hence, various methods have been studied and applied to analyze the GI tract from different perspectives. These include evaluations by endoscopy, ultrasound, magnetic resonance imaging (MRI), scintigraphy and various biomagnetic techniques. Two of the parameters that are assessed to aid in the diagnosis of gastric disorders are gastric motility and emptying. This work discusses three different techniques used to evaluated the GI lack of ionizing radiation: MRI, ultrasound, and magnetogastrography. The results obtained by each method are mentioned and compared to determine their viability and potential as complementary clinical diagnostic tests. These three alternative techniques for gastroenterology evaluations are still not routine clinical procedures and are not known by patients with gastrointestinal diseases. In authors opinion, these could bring benefits to service providers and patients themselves.
\end{abstract}

\section{Introduction}

The stomach is a J shaped organ, whose function consists in storing food, and secreting various enzymes and mucus to dosify its luminal content and produce gastric juice. Later, the gastric juice is mixed with the stored food by contractions in the smooth muscles of the stomach. The result of this mixing process, known as chyme, is then passed into the duodenum to continue digestion in the small intestine. Moreover, these functions, gastric emptying and motility, can be observed and evaluated most commonly by scintigraphy, the current gold standard [1]. However, some downsides to this technique include the high doses of ionizing radiation needed and the possible miscalculation of the gastric content due to a limited field of view since only a single projection of the stomach can be analyzed.

\section{Non-ionizing techniques for gastrointestinal evalua- tions}

There are non-invasive alternatives to scintigraphy to evaluate GI function. Some of these include evaluation by ultrasound, magnetic resonance imaging (MRI), electrogastrography (EGG), and magnetogastrography (MGG). The later two are used to obtain a bioelectric and biomagnetic analysis respectively.

As far as it is known, the first measurements of biomagnetic fields from human subjects were reported in the pioneering work by Baule and McFee (1963) [2] who recorded the magnetic field of the human heart. A biomagnetic analysis is a non-invasive alternative when looking to extract information regarding the functionality of organs. In particular, the evaluation of the gastric emptying rate, which can be studied by biomagnetic techniques, is used as an indicator for several gastrointestinal disorders such as functional dyspepsia [3, 4], diabetic gastroparesis [5], among many others.

The electrogastrography (EGG) technique consists in recording the gastric electrical activity through electrodes. These electrodes can be placed either on the abdomen or on the serosal wall (serosal electrogastrogam). In [3], measurements by Magnetogastrography (MGG) in patients with functional dyspepsia were carried out. The evaluation of the stomach's mechanical activity was possible to assess the reproducibility of the gastric emptying half-time by repeating the measurements three times, once per weeks, on each patient. MGG was demonstrated to be an appropriate method to measure the half-time of gastric emptying. It is important to emphasize that this biomagnetic modality is an easy non-invasive technique for gastroenterology research, making it a feasible method that can also be used in females

Correspondence to: Teodoro Córdova-Fraga, Departamento de Ingeniería Física-DCI, Universidad de Guanajuato campus León Loma del Bosque N. 103, Lomas del Campestre, 37150 León, GTO, Mexico, E-mail: theo@dci.ugto.mx

Key words: GI System, peristaltic, gastric emptying

Received: May 20, 2017; Accepted: June 05, 2017; Published: June 08, 2017 
during pregnancy due to the lack of ionizing radiation during this procedure.

A bioelectric and biomagnetic analysis of gastric motility creates a relationship between gastrointestinal disorders and abnormalities in the electrogastric activity [7]. It is well known that the electrical activity regulating the peristaltic movements takes place in the gastric smooth muscle. This myoelectric gastric activity has a period of 20 s with a frequency of $50 \mathrm{mHz}$, producing an electric potential of $0.1 \mathrm{mV}$ on the skin surface [8], and thus produces a magnetic field that surrounds the abdomen of approximately a few pico-Teslas. Models of this magnetic field suggest that the current density over the gastric smooth muscle is about $50 \mathrm{~mA} / \mathrm{m} 2$, implying that EGG and MGG are viable methods to study the gastric emptying rate. It is important to note though, that magnetic signals are less influenced by the tissues conductivity than the electrical signals. The signal analysis for both techniques have been developed mainly in the frequency domain by the Fast Fourier Transform (FFT) or by an adapted spectral analysis. Nevertheless, an analysis by FFT requires long-duration signals resulting in an inaccuracy at low frequencies which is why adaptive methods or an estimation of an autoregression can provide greater precision.

An alternate technique to indirectly assess gastric emptying is by ultrasound [9]. Movements in the gastric wall are evaluated, and even though a simultaneous measurement of the proximal and distal stomach is complex, this method enables to also study the effects of drugs or diseases in the gastric motility [10]. Magnetic Resonance Imaging (MRI) is yet another alternative method that records simultaneously the gastric motility and emptying $[11,12,13]$. Furthermore, both MRI and ultrasound images can be enhanced with contrast agents and facilitate determination of physiological functions such as the rate of gastric emptying. Most common contrast agents include nonparamagnetic contrast agents such as oil emulsions or those based on Gadolinium (GD-DOTA) [14], as well as some natural contrast agents.

\section{Magnetogastrography}

MGG records the magnetic field resulting from the electrogastric activity through a magnetometer. Particularly, a set of Helmholtz coils and fluxgate sensors are used to obtain MGG signals without the need of magnetically shielded room.

A protocol has been established to conduct MGG tests and states that the patient must ingest semi-solid food that has been previously validated [15], such as an egg and slice of sandwich bread with $3 \mathrm{gr}$ of magnetite particles (diameter ranging from $55 \mu \mathrm{m}$ to $125 \mu \mathrm{m}$ ) previously diluted into these. Half an hour later after ingestion, the patient is placed in a supine position along the axes of the Helmholtz coil system, as to ensure the stomach's closest proximity to the sensors [16]. A series of measurements are completed in 90 minutes, each consisting in magnetizing the magnetite particles through a series of magnetic pulses. These are generated by a discharge from a capacitor bank throughout the coil system, resulting in a $32 \mathrm{mT}$ pulsed magnetic field. This field is sufficient for the stomach to behave as a magnet for five minutes. The data acquisition is generally performed in LabVIEW and the process is repeated every 15 minutes [17], for a total time of 90 minutes.

The reproducibility of MGG has been assessed by measuring healthy men and women [16] as well as patients with functional dyspepsia [2]. Furthermore, the influence of emotions such as stress, anxiety, and depression, on the half time of gastric emptying and intestinal transit time have also been studied [17]. These emotions have shown to increase the gastric emptying time and particularly, in patients with dyspepsia, stress is the best indicator of a delayed emptying.

Various Bland Altman analysis have compared MGG with the gold standard, scintigraphy, and have shown a correlation between both measurements. The results reported that $70 \%$ of the measurements fell within a standard deviation. Additionally, a reliability greater than .80 with a sensitivity of .67 were obtained $[18,19]$.

MGG evaluates the half time of gastric emptying, making it an ideal technique to analyze gastroparetic patients, including diabetics, as well as healthy people. It achieves this without ionizing radiation unlike the gold standard, scintigraphy. Accordingly, evaluating gastrointestinal functions by MGG make this a clinically relevant tool to diagnose gastric motility dysfunctions [20,21]. Nonetheless, more studies regarding the assimilation of the magnetite particles that were ingested [15] as well as the absorption of these by the human body are needed to dismiss any health risks.

\section{Magnetic resonance imaging on the gastrointestinal system}

An image obtained by MRI is essentially a map of the distribution of water. The water density fluctuations in different tissues are compensated by manipulating $\mathrm{T} 1, \mathrm{~T} 2$, and/or by implementing an intravenous or oral contrast agent. Therefore, MRI has been proposed to study functional gastric disorders with the benefit of not needing oral intubation and a lack of ionizing radiation, offering a powerful tool to evaluate the gastric motility an emptying non-invasively. Despite MRI currently being a primary clinical imaging modality, its application as an alternative method to treat GI disorders is relatively new and still controversial.

Currently, MRI machines are widely available and are being constantly upgraded, yet spatial resolution for GI images has not improved significantly. Moreover, the application of MRI in the evaluation of GI has been limited by several problems such as, the peristalsis, respiratory and cardiac flow as well as the lack of a proper oral contrast agent [22]. Thus, to enhance GI images oral contrast agents for MRI of the gastrointestinal tract are implemented and suggest a uniform digestive distribution, a contrast independent of $\mathrm{pH}$, non-toxicity, non-peristaltic stimulus, and a reasonable price [22]. Most authors have used Gd-DOTA due to its stability in the gastric environment. Additionally, Gd-DOTA adheres relatively well to liquids and solids since it is not easily absorbed in the GI tract, making this an excellent choice as a contrast agent [13]. Also, by adopting certain MRI techniques (rapid and standard), peristalsis is no longer an MRI artifact, enabling a complete representation of the stomach's volume and the gastric peristalsis with sufficient temporal and spatial resolution [23].

Feinle et al., states that MRI has been utilized frequently to study the gastro-physiology and related diseases. In most cases, the patient is placed on the MRI scanner (1.5 $\mathrm{T}$ or greater, with a slight rise of $30^{\circ}-45^{\circ}$ ) after a fasting period as shown in figure 1. Furthermore, MRI has evolved from an alternative method to assess the gastric functions in healthy subjects into a method that can also be used to study the pharmaceutical effects on patients with functional dyspepsia as well as other functional GI disorders. Various studies and small clinics that have tried this diagnostic technique, have proven that MRI can assess both gastric motility and emptying.

In the work of Zwart-2010, a 3D scan was carried out to analyze the gastric emptying. In this case, the stomach was included in the total 


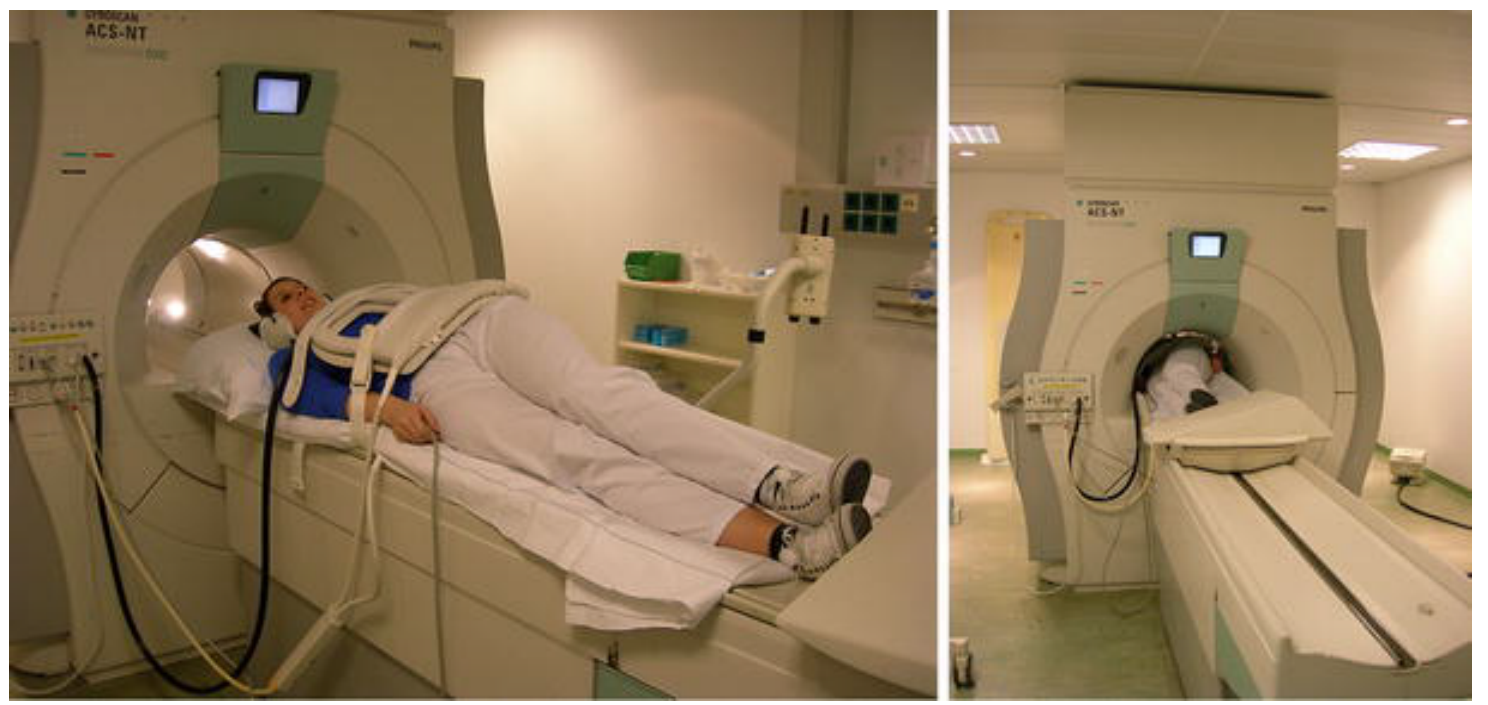

Figure 1. Patient positioning on the MRI scanner for the evaluation of gastric physiology [12]

volume acquisition by applying 0.5 to $1.0 \mathrm{~cm}$ cuts with a $1.5 \mathrm{~T}$ MRI machine. More comparative studies are needed where the protocol used to obtain images from a healthy patient in fastening, is also used to obtain images with an added oral contrast agent.

Magnetic resonance enterography has been proven to play a crucial role to determine the extension [24], disease activity, and the presence of any complication without utilizing ionizing radiation. This technique is particularly adequate to assess Crohn's disease which is mostly manifested in the young population.

Overall, by implementing the right oral contrast agent and taking advantage of the relaxation times T1 and T2, MRI is of great utility to study the GI tract. In addition, its main assets include better analysis of the tissue contrast with a great distinction between the edema fluid and the submucosal layer, multiplanar capability, multiparameter analysis, the opportunity to obtain functional information (motility, perfusion, diffusion), and its non-invasiveness (non-ionizing radiation).

\section{Ultrasound of the gastrointestinal system}

The digestive tract has for a long time been considered unsuitable for exploration by ultrasound, even though the first study showing the potential effectiveness of this technique for bowel examination dates back to the 1970s [25]. Indeed, the wall layers and histology are not exactly matching, and the resulting ultrasonography image is not of "the tissue", but instead rises from the resolution of different interfaces and reverberation artefacts [26]. Nonetheless, measurements taken with high frequency probes and with the use of contrast agents show thinner wall thickening [27].

Ultrasonography is a non-invasive, inexpensive diagnostic test with a good inter-observer agreement compared to scintigraphy [28], that provides real-time structural and functional information regarding most parameters of gastric motility [29]. Up to $50 \%$ of diabetic patients have altered gastric emptying and the predictive value of clinical symptoms is low [30]. Some studies have determined that solid particles remain in the stomach after a normal fasting period, [31] but clinical practice does not suggest an increased risk. [32]. Delayed gastric emptying has traditionally been an important preoperative factor to consider even before elective surgery, but more recent studies have shown that obese patients usually have a normal preoperative gastric volume [33]. Other applications such as the possibilities of utilizing physical influences such as magnetic field, ultrasound or light for drug delivery have been described in the work of Antipina MN, Sukhorukov GB [34].

Generally, no specific patient preparation is necessary. However, a fasting for a period prior to the examination will minimize the fluid and air present in the stomach and small intestine, resulting in better quality images [35]. The digestive system is one of the most common encroachment sites of amyloidosis, and it was reported that the gastrointestinal tract was affected in approximately $50-70 \%$ of cases [36].

\section{Discussion}

Physiology of the gastrointestinal tract is of great interest because it increases the knowledge of disorders and pathologies of the system. In particular, to diagnose functional dyspepsia, the gastric motility and emptying rates are vastly studied and analyzed. Though these can be influenced by several parameters due to the complexity of the digestive system, the physical and chemical properties of the ingested food as well as the peristaltic movements, which are regulated by an autonomous electrophysiology system, have a major role on the gastric motility and emptying rates. These factors have been employed to further develop techniques to assess these without using ionizing radiation, unlike the gold standard: scintigraphy. Furthermore, the alternative methods can be adjusted to the patient's needs. For example, claustrophobic patients that cannot tolerate MRI could choose to be tested by ultrasound or MGG. It is important to note that the last is still in a testing phase, though so far studies have proven that the biomagnetic analysis reveals significant information on the gastrophysiology. Consequently, MRI and ultrasound are the most adequate techniques to perform an analysis on gastric motility and emptying and can complement other studies to further improve clinical diagnosis and prognosis of GI disorders.

\section{Acknowledgments}

Authors thank the partial funding of the project from DAIP2016/5923.

\section{References}

1. Benini L, Sembenini C, Heading RC, Giorgetti PG, Montemezzi S et al. (1999) Simultaneus measurement of gastric emptying of a solid meal by ultrasound and by Scintigraphy. Am J Gastroenterol 94: 2861-2865. [Crossref] 
2. Baule G M and McFee R (1963) Detection of the magnetic ?eld of the heart. Am. Heart J 66: 95-96.

3. De la Roca-Chiapas JM, Cordova-Fraga T, Zarate A et al. Magnetogastrography in patients with functional dyspepsia. Biomagnetism: in interdisciplinary research and exploration. Proceedings of the 16th international conference on biomagnetism. Hokkaido University Press, Japan, pp 15-17, (2008).

4. Haans JJ, Masclee AA (2007) Review article: The diagnosis and management of gastroparesis. Aliment Pharmacol Ther 26 Suppl 2: 37-46. [Crossref]

5. Anahi Perlas, W. S. Vincent Chan, Catalin M. Lupu, Nicholas Mitsakakis, and Anthony Hanbidge. (2009) Ultrasound Assessment of Gastric Content and Volume, Anesthesiology, 111(1)

6. Schmid MR, Hany TF, Knesplova L, Schlumpf R, Debatin JF (1999) 3D MR gastrography: exoscopic and endoscopic analysis of the stomach. Eur Radiol 9: 73-77. [Crossref]

7. A. Irimia, W. O. Richards and L. A. Bradshaw (2006) Magnetogastrographic detection of gastric electrical response activity in humans. Physiscs in Medicine and Biology 51: 1347-1360. [Crossref]

8. Van de Putte P, Perlas A2 (2014) Ultrasound assessment of gastric content and volume. Br J Anaesth 113: 12-22. [Crossref]

9. Bateman DN, Whittingham TA (1982) Measurement of gastric emptying by real-time ultrasound. Gut 23: 524-527. [Crossref]

10. Schwizer W, Fraser R, Borovicka J, Crelier G, Boesiger P, et al. (1994) Measurement of gastric emptying and gastric motility by magnetic resonance imaging (MRI). Dig Dis Sci 39: 101S-103S. [Crossref]

11. de Zwart IM, de Roos A (2010) MRI for the evaluation of gastric physiology. Eur Radiol 20: 2609-2616. [Crossref]

12. Schwizer W, Fraser R, Maecke H, Siebold K, Funck R et al. (1994) Gd-DOTA as a gastrointestinal contrast agent for gastric emptying measurements with MRI. Magn Reson Med 31: 388-393. [Crossref]

13. P. Kunz, C. Feinle, W. Schwizer, M. Fried and P. Boesiger (1999) Assessment of gastric motor function during the emptying of solid and liquid meals in humans by MRI. J Magn Reson Imaging 9: 75-80 [Crossref]

14. Teodoro CF, José María De la Roca-Chiapas, Silvia Solís, Modesto Sosa, Jesús BernalAlvarado Enedino Hernández et al. (2008) Gender difference in the gastric emptying measured by magnetogastrography using a semi-solid test meal. Acta Gastroenterol Latinoam 38: 240-245 [Crossref]

15. Reynaga-Ornelas MG, De la Roca-Chiapas JM, Cordova -Fraga T, Bernal JJ, Sosa M (2008) Solid Test meal to measure the gastric emptying with Magnetogastrography. AIP Conferences Procceding vol 1032: 246-248

16. José Ma. De la Roca-Chiapas, Silvia Solís-Ortiz, Martha Fajardo-Araujo, Modesto Sosa, Teodoro Córdova-Fraga (2010) Stress profile, coping style, anxiety, depression, and gastric emptying as predictors of functional dyspepsia: A case-control study. $J$ Psychosom Res 68: 73-81 [Crossref]

17. J. M. De la Roca-Chiapas, T. Cordova-Fraga, G. Reynaga, S. Solorio, M. Sosa, A. E. Rivera-Cisneros, J. J. Bernal.M. Vargas-Luna. (2010) Scintigraphy vs. mechanical magnetogastrography:gastric emptying analysis. Med Biol Eng Comput. 48: 727-9. [Crossref]

18. De la Roca-Chiapas JM and Cordova- Fraga T. (2015) Biomagnetic techniques for evaluating gastric emptying, peristaltic contraction and transit time. Articulo Editorial en la Revista World Journal of Gastrointestinal Pathophysiology 2: 65-71
19. Estombelo-Montesco CA, de Araujo DB, Silva Filho AC, Moraes ER, Barros AK, et al. (2007) Dependent component analysis for the magnetogastrographic detection of human electrical response activity. Physiol Meas 28: 1029-1044. [Crossref]

20. Moraes ER, Toncon LE, Baffa O, Oba-Kunyioshi AS, Wakai R, Leuthold A Adaptative, autoregressive spectral estimation for analysis of electrical signals of gastric origin, Physiological Measurement, Vol. 24, 2003.

21. De la Roca-Chiapas JM, Cordova T, Hernandez E, Solorio S, Solis S, Sosa M Magnetogastrography (MGG) reproducibility assessments of gastric emptying on healthy subjects. Physiol Meas 28: 175-183, (2007).

22. Teodoro Cordova-Fraga, Modesto Sosa, Martha Alicia Hernandez-Gonzalez, Jose Antonio Reyes-Aguilera, Sergio Solorio, Chystian Ramirez, Evelia Bautista-Flores Guadalupe Reynaga, Mario Avila-Rodriguez and Jose Maria De la Roca-Chiapas. Medlar (Achras Sapota L.) as Oral Contrast Agent or MRI of the Gastrointestinal Tract. Appl Magn Reson. 42: 161-167 (2012).

23. de Zwart IM, de Roos A (2010) MRI for the evaluation of gastric physiology. Eur Radiol 20: 2609-2616. [Crossref]

24. Mantarro A, Scalise P1, Guidi E1, Neri E1 (2017) Magnetic resonance enterography in Crohn's disease: How we do it and common imaging findings. World J Radiol 9: 46-54. [Crossref]

25. Hauser JB, Stanley RJ, Geisse G (1974) The ultrasound findings in an obstructed afferent loop. J Clin Ultrasound 2: 287-289. [Crossref]

26. Nylund K, Leh S, Immervoll H, Matre K, Skarstein A et al. (2008) Crohn's disease: Comparison of in vitro ultrasonographic images and histology. Scand J Gastroenterol 43: 719-726, [Crossref]

27. Nylund K, Hausken T, Odegaard S, Eide GE, Gilja OH (2012) Gastrointestinal wall thickness measured with transabdominal ultrasonography and its relationship to demographic factors in healthy subjects. Ultraschall Med 33: E225-232. [Crossref]

28. Irvine EJ, Tougas G, Lappalainen R, Bathurst NC (1993) Reliability and interobserver variability of ultrasonographic measurement of gastric emptying rate. Dig Dis Sci 38: 803-810. [Crossref]

29. Gilja OH1 (2007) Ultrasound of the stomach--the EUROSON lecture 2006. Ultraschall Med 28: 32-39. [Crossref]

30. Darwiche G, Bjo"rgell O, Thorsson O, Alme'r LO. (2003) Correlation between simultaneous scintigraphic and ultrasonographic measurement of gastric emptying in patients with type 1 diabetes mellitus. J Ultrasound Med 22: 459 - 466 [Crossref]

31. Ishihara H, Singh H, Giesecke AH (1994) Relationship between diabetic autonomic neuropathy and gastric contents. Anesth Analg 78: 943-947. [Crossref]

32. JellishWS, KarthaV, Fluder E, Slogoff S. (2005) Effect of metoclopramide on gastric fluid volumes in diabetic patients who have fasted before elective surgery. Anesthesiology 102: 904-909 [Crossref]

33. Donohoe CL, Feeney C, Carey MF, Reynolds JV. (2011) Perioperative evaluation of the obese patient. J Clin Anesth 23: 575-86.

34. Antipina MN, Sukhorukov GB. Remote control over guidance and release properties of composite polyelectrolyte based capsules. Adv Drug Deliv Rev 63: 716-729 (2011)

35. Nylund K, Ødegaard S, Hausken T, Folvik G, Lied GA, et al. (2009) Sonography of the small intestine. World J Gastroenterol 15: 1319-1330. [Crossref]

36. Nfoussi H, Chelly I, Bellil K, Kchir N, Haouet S, et al. (2010) [Pseudo tumoral gastric amyloidosis: a case report]. Tunis Med 88: 670-673. [Crossref]

Copyright: (C2017 Córdova-Fraga T. This is an open-access article distributed under the terms of the Creative Commons Attribution License, which permits unrestricted use, distribution, and reproduction in any medium, provided the original author and source are credited. 